\title{
A Discrete Version of Plane Wave Solutions of the Dirac Equation in the Joyce Form
}

\author{
Volodymyr Sushch*®
}

\author{
Communicated by Vladislav Kravchenko
}

\begin{abstract}
We construct a discrete version of the plane wave solution to a discrete Dirac-Kähler equation in the Joyce form. A geometric discretisation scheme based on both forward and backward difference operators is used. The conditions under which a discrete plane wave solution satisfies a discrete Joyce equation are discussed.
\end{abstract}

Mathematics Subject Classification. Primary 39A12; Secondary 81Q05, 39A70.

Keywords. Dirac-Kähler equation, Joyce equation, Discrete models, Geometric discretisation, Difference equations, Plane wave solution.

\section{Introduction}

Discrete models of Dirac type equations based on the Dirac-Kähler formulation have been of interest recently from both the applied and the theoretical point of view. In the Dirac-Kähler approach, a discretisation scheme is geometric in nature and rests upon the use of the differential forms calculus. This means that the geometric properties and the algebraic relationships between the differential, the exterior product, and the Hodge star operator are expected to be captured in the case of their discrete counterparts. This work is a continuation of that studied in the papers [15-19]. In this paper, we are mainly interested in a discrete model in which both forward and backward differences operators are used. This model relies on the use of a geometric discretisation scheme proposed in [15] and a discrete Clifford calculus framework on discrete forms described in [18]. For a review of discrete Clifford calculus frameworks on lattices, we refer the reader to $[3,5-7,13,20]$. Our purpose here is to construct a discrete version of the plane wave solution to a discrete Dirac-Kähler equation in the Joyce form. We first briefly

\footnotetext{
${ }^{*}$ Corresponding author
} 
review some definitions and basic facts on the Dirac-Kähler equation $[12,14]$ and the Dirac equation in the spacetime algebra $[8,9]$. Let $M=\mathbb{R}^{1,3}$ be Minkowski space. Denote by $\Lambda^{r}(M)$ the vector space of smooth differential $r$-forms, $r=0,1,2,3,4$. We consider $\Lambda^{r}(M)$ over $\mathbb{C}$. Let $\omega, \varphi \in \Lambda^{r}(M)$. The inner product is defined by

$$
(\omega, \varphi)=\int_{M} \omega \wedge * \bar{\varphi},
$$

where $\wedge$ is the exterior product, $\bar{\varphi}$ denotes the complex conjugate of the form $\varphi$ and $*$ is the Hodge star operator $*: \Lambda^{r}(M) \rightarrow \Lambda^{4-r}(M)$ with respect to the Lorentz metric. Let $d: \Lambda^{r}(M) \rightarrow \Lambda^{r+1}(M)$ be the exterior differential and let $\delta: \Lambda^{r}(M) \rightarrow \Lambda^{r-1}(M)$ be the formal adjoint of $d$ with respect to (1.1). We have $\delta=* d *$. Denote by $\Lambda(M)$ the set of all differential forms on $M$. We have $\Lambda(M)=\Lambda^{0}(M) \oplus \Lambda^{1}(M) \oplus \Lambda^{2}(M) \oplus \Lambda^{3}(M) \oplus \Lambda^{4}(M)$. Let $\Omega \in \Lambda(M)$ be an inhomogeneous differential form, i.e.,

$$
\Omega=\sum_{r=0}^{4} \stackrel{r}{\omega}
$$

where $\stackrel{r}{\omega} \in \Lambda^{r}(M)$. The Dirac-Kähler equation for a free electron is given by

$$
i(d+\delta) \Omega=m \Omega,
$$

where $i$ is the usual complex unit and $m$ is a mass parameter. It is easy to show that Eq. (1.3) is equivalent to the set of equations

$$
\begin{aligned}
i \delta \dot{\omega}^{1} & =m \stackrel{0}{\omega}, \\
i(d \stackrel{0}{\omega}+\delta \stackrel{2}{\omega}) & =m \stackrel{1}{\omega}, \\
i(d \stackrel{1}{\omega}+\delta \stackrel{3}{\omega}) & =m \stackrel{2}{\omega}, \\
i(d \stackrel{2}{\omega}+\delta \stackrel{4}{\omega}) & =m \stackrel{3}{\omega}, \\
i d^{3} & =m \stackrel{4}{\omega} .
\end{aligned}
$$

The operator $d+\delta$ is the analogue of the gradient operator in Minkowski spacetime

$$
\nabla=\sum_{\mu=0}^{3} \gamma_{\mu} \partial^{\mu}, \quad \mu=0,1,2,3,
$$

where $\gamma_{\mu}$ is the Dirac gamma matrix. If one think of $\left\{\gamma_{0}, \gamma_{1}, \gamma_{2}, \gamma_{3}\right\}$ as a vector basis in spacetime, then the gamma matrices $\gamma_{\mu}$ can be considered as generators of the Clifford algebra of signature $(1,3)$ denoted by $\mathcal{C} \ell(1,3)$ $[1,2]$. Hestenes [9] calls this algebra the spacetime algebra. It is known that an inhomogeneous form $\Omega$ can be represented as an element of $\mathcal{C} \ell(1,3)$. Then the Dirac-Kähler equation can be written as the algebraic equation

$$
i \nabla \Omega=m \Omega,
$$

where $\Omega=\Omega(x) \in \mathcal{C} \ell(1,3)$ is a multivector function. Equation (1.5) is equivalent to the four Dirac equations (traditional column-spinor equations) for 
a free electron. Let $\mathcal{C} \ell^{e v}(1,3)$ be the even subalgebra of the algebra $\mathcal{C} \ell(1,3)$. Consider the equation

$$
i \nabla \Omega^{e v}=m \Omega^{e v} \gamma_{0}
$$

where $\Omega^{e v}=\Omega^{e v}(x) \in \mathcal{C} \ell^{e v}(1,3)$ is an even multivector function. In [10], this equation is called the "generalized bivector Dirac equation". Following Baylis [2], we call Eq. (1.6) the Joyce equation. This equation is equivalent to two copies of the usual Dirac equation. For a deeper discussion of the equivalence of Dirac formulations, we refer the reader to [11]. Equation (1.6) admits the plane wave solution of the form

$$
\Phi_{ \pm}=A \exp ( \pm i p \cdot x)
$$

where $A \in \mathcal{C} \ell^{e v}(1,3)$ is a constant element, $p=\left\{p_{0}, p_{1}, p_{2}, p_{3}\right\}$ is a fourmomentum and $p \cdot x=p_{0} x^{0}-p_{1} x^{1}-p_{2} x^{2}-p_{3} x^{3}$ (see [2] for more details).

It should be noted that the graded algebra $\Lambda(M)$ endowed with the geometric product is an example of a Clifford algebra. In this case the basis covectors $e^{\mu}=d x^{\mu}$ are considered as generators of the Clifford algebra. Let $\Lambda^{e v}(M)=\Lambda^{0}(M) \oplus \Lambda^{2}(M) \oplus \Lambda^{4}(M)$. Denote by $\Omega^{e v}$ the even part of the form (1.2). Then Eq. (1.6) can be rewritten in terms of inhomogeneous forms as

$$
i(d+\delta) \Omega^{e v}=m \Omega^{e v} e^{0}, \quad \Omega^{e v} \in \Lambda^{e v}(M) .
$$

We call this equation the Dirac-Kähler equation in the Joyce form.

In this paper, we focus on the construction of a discrete version of the plane wave solution (1.7) for a discrete counterpart of Eq. (1.8). In [18], the same problem was considered by using a discretisation scheme based on a combinatorial double complex construction. Such a discretisation method was also applied in the paper [17] to construct a similar solution for a discrete Dirac-Kähler equation in the Hestenes form. However, the double complex construction generates difference operators of the forward type only and it is not enough to obtain an exact geometric counterpart of the Clifford algebra $\mathcal{C} \ell(1,3)$. In $[20]$, it has been shown that a discrete model that uses only one type of difference operators produces a deformation of the underlying Clifford algebra in the continuum limit. The fact that both forward and backward differences on the lattice are needed is well-known [14]. To avoid this problem we modify the discretisation scheme and define the main operations of the discrete model without using the double complex construction. As a new result, we announce here the construction of a discrete plane wave solution based on both forward and backward difference operators. This suggests that such a discrete model is more close to the continuum counterpart as the comparison with $[17,18]$. The clear intrinsically defined difference representations of the main equations and an explicit formula for the general solution are an adventure of our discretisation method. One more motivation for applying this approach is that it is formulated without using a matrix representation of Clifford algebra. 


\section{Combinatorial Model and Difference Operators}

A combinatorial model of Minkowski space and a discretisation scheme are adopted from [15]. The idea of such geometric discretization goes back at least as far as [4]. For the convenience of the reader, we briefly repeat the relevant material from [15] without proofs, thus making our presentation selfcontained. Following [4], let the tensor product $C(4)=C \otimes C \otimes C \otimes C$ of a 1-dimensional complex be a combinatorial model of Euclidean space $\mathbb{R}^{4}$. The 1-dimensional complex $C$ is defined in the following way. Introduce the sets $\left\{x_{\kappa}\right\}$ and $\left\{e_{\kappa}\right\}, \kappa \in \mathbb{Z}$. Let $C^{0}$ and $C^{1}$ be the free abelian groups of 0 -dimensional and 1-dimensional chains generated by $\left\{x_{\kappa}\right\}$ and $\left\{e_{\kappa}\right\}$. The free abelian group is understood as the direct sum of infinity cyclic groups generated by $\left\{x_{\kappa}\right\},\left\{e_{\kappa}\right\}$. The boundary operator $\partial: C^{0} \rightarrow 0, \quad \partial: C^{1} \rightarrow C^{0}$ is given by

$$
\partial x_{\kappa}=0, \quad \partial e_{\kappa}=x_{\kappa+1}-x_{\kappa} .
$$

The definition is extended to arbitrary chains by linearity. The direct sum $C=C^{0} \oplus C^{1}$ with the boundary operator $\partial$ defines the 1-dimensional complex. It is known that a free abelian group is an abelian group with basis. One can regard the sets $\left\{x_{\kappa}\right\},\left\{e_{\kappa}\right\}$ as sets of basis elements of the groups $C^{0}$ and $C^{1}$. Geometrically we can interpret the 0 -dimensional basis elements $x_{\kappa}$ as points of the real line and the 1 -dimensional basis elements $e_{\kappa}$ as open intervals between points, i.e., $e_{\kappa}=\left(x_{\kappa}, x_{\kappa+1}\right)$. We call the complex $C$ a combinatorial real line. Every basis element of $C(4)$ can be written as

$$
s_{k}=s_{k_{0}} \otimes s_{k_{1}} \otimes s_{k_{2}} \otimes s_{k_{3}},
$$

where $s_{k_{\mu}}$ is either $x_{k_{\mu}}$ or $e_{k_{\mu}}$ and $k=\left(k_{0}, k_{1}, k_{2}, k_{3}\right), k_{\mu} \in \mathbb{Z}$. Or more precisely, denote by $s_{k}^{(r)}$ the $r$-dimensional basis element of $C(4)$, where the superscript $(r)$ means that the product (2.2) contains exactly $r, r=$ 0,1,2,3,4, 1-dimensional elements $e_{k_{\mu}}$ and $4-r$ 0-dimensional elements $x_{k_{\mu}}$, and indicates also a position of $e_{k_{\mu}}$ in $s_{k}^{(r)}$. Let $C(p)$ is the tensor product of $p$ factors of $C=C(1), p=1,2,3$. The definition (2.1) of $\partial$ is extended to an arbitrary basis element of $C(4)$ by induction on $p$. Suppose that the boundary operator has been defined for any basis element $s_{k} \in C(p)$. Then we introduce it for the basis element $s_{k_{\mu}} \otimes s_{k} \in C(p+1)$ by the rule

$$
\partial\left(s_{k_{\mu}} \otimes s_{k}\right)=\partial s_{k_{\mu}} \otimes s_{k}+Q\left(k_{\mu}\right) s_{k_{\mu}} \otimes \partial s_{k},
$$

where $s_{k_{\mu}} \in C$ and $Q\left(k_{\mu}\right)$ is equal to +1 if $s_{k_{\mu}}=x_{k_{\mu}}$ and to -1 if $s_{k_{\mu}}=e_{k_{\mu}}$. The operation (2.3) is linearly extended to arbitrary chains.

Let us construct the dual complex to $C(4)$ which will play a role of a discrete counterpart of the space $\Lambda(M)$. Denote by $K(4)=K \otimes K \otimes K \otimes K$ a cochain complex with complex coefficients, where $K$ is the 1-dimensional complex generated by 0 - and 1-dimensional basis elements $x^{k_{\mu}}$ and $e^{k_{\mu}}, k_{\mu} \in$ $\mathbb{Z}$, respectively. Then an arbitrary $r$-dimensional basis element of $K(4)$ can be written as $s_{(r)}^{k}=s^{k_{0}} \otimes s^{k_{1}} \otimes s^{k_{2}} \otimes s^{k_{3}}$, where $s^{k_{\mu}}$ is either $x^{k_{\mu}}$ or $e^{k_{\mu}}$, $\mu=0,1,2,3$ and $k=\left(k_{0}, k_{1}, k_{2}, k_{3}\right)$. Again, the symbol $(r)$ contains the whole required information about the number and position $e^{k_{\mu}} \in K$ in $s_{(r)}^{k} \in$ $K(4)$. Denote by $K^{r}(4)$ the set of all $r$-dimensional cochains. Then $K(4)$ can 
be expressed by $K(4)=K^{0}(4) \oplus K^{1}(4) \oplus K^{2}(4) \oplus K^{3}(4) \oplus K^{4}(4)$ and for $\stackrel{r}{\omega} \in K^{r}(4)$ we have

$$
\stackrel{r}{\omega}=\sum_{k} \sum_{(r)} \omega_{k}^{(r)} s_{(r)}^{k},
$$

where $(r)$ ranges over all increasing subsets of length $r$ from $\{0,1,2,3\}, k=$ $\left(k_{0}, k_{1}, k_{2}, k_{3}\right)$ is a multi-index, and $\omega_{k}^{(r)} \in \mathbb{C}$.

As in [4], for any basis element $s^{k} \in K(4)$ the pairing with a basis element $\varepsilon_{k} \in C(4)$ is defined by

$$
\left\langle\varepsilon_{k}, s^{k}\right\rangle= \begin{cases}0, & \varepsilon_{k} \neq s_{k} \\ 1, & \varepsilon_{k}=s_{k}\end{cases}
$$

for each $k=\left(k_{0}, k_{1}, k_{2}, k_{3}\right), k_{\mu} \in \mathbb{Z}$. The operation $(2.5)$ is linearly extended to arbitrary chains and cochains.

The coboundary operator $d^{c}: K^{r}(4) \rightarrow K^{r+1}(4)$ is generated by the boundary operator $\partial$ according to the rule

$$
\langle\partial a, \stackrel{r}{\omega}\rangle=\left\langle a, d^{c} \stackrel{r}{\omega}\right\rangle
$$

where $a \in C(4)$ is an $r+1$ dimensional chain. By definition $d^{c} \stackrel{4}{\omega}=0$ and $d^{c} d^{c} \stackrel{r}{\omega}=0$ for any $r$. The complex of cochains $K(4)$ with the operator $d^{c}$ defined in it is the dual of $C(4)$. Assume that $K(4)$ is a discrete analogue of $\Lambda(M)$. In what follows we call cochains forms or discrete forms to emphasize their relationship with differential forms. Then the operator $d^{c}$ is an analog of the exterior differential. Note that relation (2.6) is an analog of the Stokes theorem.

It is convenient to introduce the shift operators $\tau_{\mu}$ and $\sigma_{\mu}$ in the set of indices by

$$
\tau_{\mu} k=\left(k_{0}, \ldots k_{\mu}+1, \ldots k_{3}\right), \quad \sigma_{\mu} k=\left(k_{0}, \ldots k_{\mu}-1, \ldots k_{3}\right), \quad \mu=0,1,2,3 .
$$

Let the difference operators $\Delta_{\mu}^{+}$and $\Delta_{\mu}^{-}$be defined by

$$
\begin{aligned}
& \Delta_{\mu}^{+} \omega_{k}^{(r)}=\omega_{\tau_{\mu} k}^{(r)}-\omega_{k}^{(r)}, \\
& \Delta_{\mu}^{-} \omega_{k}^{(r)}=\omega_{k}^{(r)}-\omega_{\sigma_{\mu} k}^{(r)},
\end{aligned}
$$

where $\omega_{k}^{(r)} \in \mathbb{C}$ is a component of $\stackrel{r}{\omega} \in K^{r}(4)$. It is clear that

$$
\Delta_{\mu}^{-} \omega_{k}^{(r)}=\Delta_{\mu}^{+} \omega_{\sigma_{\mu} k}^{(r)}, \quad \Delta_{\mu}^{+} \omega_{k}^{(r)}=\Delta_{\mu}^{-} \omega_{\tau_{\mu} k}^{(r)} .
$$

Note that it is enough to use one of the two above defined difference operators to describe discrete analogs of $d$ and $\delta$, as it is shown in [15], but in the proposed approach both forward and backward differences are needed to construct a discrete version of the plane wave solution. It is helpful here to write (2.4) more explicitly as 


$$
\begin{aligned}
& \stackrel{0}{\omega}=\sum_{k} \stackrel{0}{\omega}_{k} x^{k}, \quad \stackrel{1}{\omega}=\sum_{k} \sum_{\mu=0}^{3} \omega_{k}^{\mu} e_{\mu}^{k}, \quad \stackrel{2}{\omega}=\sum_{k} \sum_{\mu<\nu} \omega_{k}^{\mu \nu} e_{\mu \nu}^{k}, \\
& \stackrel{3}{\omega}=\sum_{k}\left(\omega_{k}^{012} e_{012}^{k}+\omega_{k}^{013} e_{013}^{k}+\omega_{k}^{023} e_{023}^{k}+\omega_{k}^{123} e_{123}^{k}\right), \quad \stackrel{4}{\omega}=\sum_{k} \stackrel{4}{\omega}_{k} e^{k},
\end{aligned}
$$

where

$$
x^{k}=x^{k_{0}} \otimes x^{k_{1}} \otimes x^{k_{2}} \otimes x^{k_{3}}, \quad e^{k}=e^{k_{0}} \otimes e^{k_{1}} \otimes e^{k_{2}} \otimes e^{k_{3}}
$$

are the 0-, 4-dimensional basis elements of $K(4)$, and $e_{\mu}^{k}, e_{\mu \nu}^{k}$ and $e_{\iota \mu \nu}^{k}$ are the 1-, 2- and 3-dimensional basis elements of $K(4)$. Here the notation $\mu<\nu$ means that the sum runs over all indices $\mu, \nu$ such that $0 \leq \mu<\nu \leq 3$.

Using (2.3), (2.6) and (2.8) we can calculate

$$
\begin{aligned}
d^{c} \stackrel{0}{\omega}= & \sum_{k} \sum_{\mu=0}^{3}\left(\Delta_{\mu}^{+} \stackrel{0}{\omega}_{k}\right) e_{\mu}^{k}, \quad d^{c} \stackrel{1}{\omega}=\sum_{k} \sum_{\mu<\nu}\left(\Delta_{\mu}^{+} \omega_{k}^{\nu}-\Delta_{\nu}^{+} \omega_{k}^{\mu}\right) e_{\mu \nu}^{k}, \\
d^{c} \stackrel{2}{\omega}= & \sum_{k}\left[\left(\Delta_{0}^{+} \omega_{k}^{12}-\Delta_{1}^{+} \omega_{k}^{02}+\Delta_{2}^{+} \omega_{k}^{01}\right) e_{012}^{k}\right. \\
& +\left(\Delta_{0}^{+} \omega_{k}^{13}-\Delta_{1}^{+} \omega_{k}^{03}+\Delta_{3}^{+} \omega_{k}^{01}\right) e_{013}^{k} \\
& +\left(\Delta_{0}^{+} \omega_{k}^{23}-\Delta_{2}^{+} \omega_{k}^{03}+\Delta_{3}^{+} \omega_{k}^{02}\right) e_{023}^{k} \\
& \left.+\left(\Delta_{1}^{+} \omega_{k}^{23}-\Delta_{2}^{+} \omega_{k}^{13}+\Delta_{3}^{+} \omega_{k}^{12}\right) e_{123}^{k}\right] \\
d^{c} \stackrel{3}{\omega}= & \sum_{k}\left(\Delta_{0}^{+} \omega_{k}^{123}-\Delta_{1}^{+} \omega_{k}^{023}+\Delta_{2}^{+} \omega_{k}^{013}-\Delta_{3}^{+} \omega_{k}^{012}\right) e^{k} .
\end{aligned}
$$

Let us now introduce a $\cup$-multiplication of discrete forms which is an analog of the exterior multiplication for differential forms. Denote by $K(p)$ the tensor product of $p$ factors of the 1-dimensional complex $K=K(1)$. For the basis elements of $K$ the $\cup$-multiplication is defined as follows

$$
x^{k_{\mu}} \cup x^{k_{\mu}}=x^{k_{\mu}}, \quad e^{k_{\mu}} \cup x^{k_{\mu}+1}=e^{k_{\mu}}, \quad x^{k_{\mu}} \cup e^{k_{\mu}}=e^{k_{\mu}}, \quad k_{\mu} \in \mathbb{Z},
$$

supposing the product to be zero in all other case. To arbitrary basis elements of $K(p), p=2,3,4$, the definition (2.14) is extended by induction on $p$ (see [4] for more details). Again, to arbitrary discrete forms the $\cup$-multiplication is extended linearly. It is important to note that the definition above is suitable to deal with a discrete version of the Leibniz rule. The following result was proven in [4, Proposition 2, p. 147].

Proposition 2.1. Let $\varphi \in K^{r}(4)$ and $\psi \in K^{q}(4)$ be arbitrary discrete forms. Then

$$
d^{c}(\varphi \cup \psi)=d^{c} \varphi \cup \psi+(-1)^{r} \varphi \cup d^{c} \psi .
$$

A discrete analogue of the Hodge star operator is one of the main distinctive features of the present discretisation scheme as compared to [17]. In [17], to define a discrete counterpart of the Hodge star the combinatorial double complex construction which is too awkward and some unclear geometrically is used. Now we define the operation $*: K^{r}(4) \rightarrow K^{4-r}(4)$ for an arbitrary basis element $s^{k}=s^{k_{0}} \otimes s^{k_{1}} \otimes s^{k_{2}} \otimes s^{k_{3}}$ by the rule

$$
s^{k} \cup * s^{k}=Q\left(k_{0}\right) e^{k} \text {, }
$$


where $Q\left(k_{0}\right)$ is equal to +1 if $s^{k_{0}}=x^{k_{0}}$ and to -1 if $s^{k_{0}}=e^{k_{0}}$, and $e^{k}$ is given by (2.10). For example, for the 1-dimensional basis elements $e_{\mu}^{k}$ we have $e_{0}^{k} \cup * e_{0}^{k}=-e^{k}$ and $e_{\mu}^{k} \cup * e_{\mu}^{k}=e^{k}$ for $\mu=1,2,3$. Here for simplicity of notation, we continue to write $*$ in the discrete case. Note that replacing $\cup$ by $\wedge$ in (2.16) gives the usual definition of the Hodge star on $\Lambda^{r}(M)$. Hence (2.16) defines a discrete analogue of $*$ which is more natural than the one defined in [17]. It is clear that the definition (2.16) preserves the Lorentz signature of metric in our discrete model. From (2.16) a more detailed calculation leads to

$$
\begin{aligned}
* x^{k} & =e^{k}, \quad * e^{k}=-x^{\tau k}, \\
* e_{0}^{k} & =-e_{123}^{\tau_{0} k}, \quad * e_{1}^{k}=-e_{023}^{\tau_{1} k}, \quad * e_{2}^{k}=e_{013}^{\tau_{2} k}, \quad * e_{3}^{k}=-e_{012}^{\tau_{3} k}, \\
* e_{01}^{k} & =-e_{23}^{\tau_{01} k}, \quad * e_{02}^{k}=e_{13}^{\tau_{02} k}, \quad * e_{03}^{k}=-e_{12}^{\tau_{03} k}, \\
* e_{12}^{k} & =e_{03}^{\tau_{12} k}, \quad * e_{13}^{k}=-e_{02}^{\tau_{13} k}, \quad * e_{23}^{k}=e_{01}^{\tau_{23} k}, \\
* e_{012}^{k} & =-e_{3}^{\tau_{012} k}, * e_{013}^{k}=e_{2}^{\tau_{013} k}, \quad * e_{023}^{k}=-e_{1}^{\tau_{023} k}, * e_{123}^{k}=-e_{0}^{\tau_{123} k}
\end{aligned}
$$

Here $\tau_{\mu \nu} k=\tau_{\mu} \tau_{\nu} k$ and $\tau_{\mu \nu \iota} k=\tau_{\mu} \tau_{\nu} \tau_{\iota} k$, where $\tau_{\mu}$ is defined by (2.7), and

$$
\tau k=\left(k_{0}+1, k_{1}+1, k_{2}+1, k_{3}+1\right) .
$$

The operation $*$ is linearly extended to arbitrary forms. It is easy to check that

$$
* * s_{(r)}^{k}=(-1)^{r+1} s_{(r)}^{\tau k},
$$

where $s_{(r)}^{k}$ is an $r$-dimensional basic element of $K(4)$. Then if we perform the operation $*$ twice on any $r$-form $\stackrel{r}{\omega} \in K(4)$, we obtain

$$
* * \stackrel{r}{\omega}=(-1)^{r+1} \sum_{k} \sum_{(r)} \omega_{k}^{(r)} s_{(r)}^{\tau k}=(-1)^{r+1} \sum_{k} \sum_{(r)} \omega_{\sigma k}^{(r)} s_{(r)}^{k},
$$

where $\tau k$ is given by $(2.21)$ and

$$
\sigma k=\left(k_{0}-1, k_{1}-1, k_{2}-1, k_{3}-1\right) .
$$

Hence the operation $(*)^{2}$ is equivalent to a shift with corresponding sign. This is slightly different from the continuum case, where applying the Hodge star operator twice leaves a differential form unchanged up to sign, i.e., $(*)^{2}= \pm 1$.

Now to introduce a discrete counterpart of the codifferential operator $\delta$ we need to construct an inner product on the space of discrete forms. Consider the 4-dimensional finite chain $e_{n} \subset C(4)$ of the form

$$
e_{n}=\sum_{k} e_{k}, \quad k_{\mu}=1,2, \ldots, n_{\mu}
$$

where $n_{\mu} \in \mathbb{N}$ is a fixed natural number for each $\mu=0,1,2,3$ and $e_{k}$ is given by $(2.10)$. In other words, the finite sum (2.23) is the set defined by a finite number of 4 -dimensional basis elements of $C(4)$. Suppose that the $r$-form (2.4) is vanished on $C(4) \backslash e_{n}$, i.e., if $k_{\mu}<1$ or $k_{\mu}>n_{\mu}$ then $\omega_{k}^{(r)}=0$ for 
any $r$ and $k$. Then for forms $\varphi, \omega \in K^{r}(4)$ of the same degree $r$ the inner product is defined by the rule

$$
(\varphi, \omega)_{e_{n}}=\left\langle e_{n}, \varphi \cup * \bar{\omega}\right\rangle,
$$

where $\bar{\omega}$ denotes the complex conjugate of the form $\omega$. For forms of different degrees the product (2.24) is set equal to zero. The definition (2.24) imitates correctly the continuum case (1.1) and the Lorentz metric structure is still captured here. Using (2.5), (2.14) and (2.17)-(2.20) we obtain

$$
\begin{aligned}
& (\stackrel{0}{\varphi}, \stackrel{0}{\omega})_{e_{n}}=\sum_{k} \stackrel{0}{\varphi}_{k} \bar{\omega}_{k}, \quad(\stackrel{4}{\varphi}, \stackrel{4}{\omega})_{e_{n}}=-\sum_{k} \stackrel{4}{\varphi}_{k} \frac{4}{\omega} \bar{\omega}_{k}, \\
& (\stackrel{1}{\varphi}, \stackrel{1}{\omega})_{e_{n}}=\sum_{k}\left(-\varphi_{k}^{0} \bar{\omega}_{k}^{0}+\varphi_{k}^{1} \bar{\omega}_{k}^{1}+\varphi_{k}^{2} \bar{\omega}_{k}^{2}+\varphi_{k}^{3} \bar{\omega}_{k}^{3}\right), \\
& (\stackrel{2}{\varphi}, \stackrel{2}{\omega})_{e_{n}}=\sum_{k}\left(-\varphi_{k}^{01} \bar{\omega}_{k}^{01}-\varphi_{k}^{02} \bar{\omega}_{k}^{02}-\varphi_{k}^{03} \bar{\omega}_{k}^{03}+\varphi_{k}^{12} \bar{\omega}_{k}^{12}+\varphi_{k}^{13} \bar{\omega}_{k}^{13}+\varphi_{k}^{23} \bar{\omega}_{k}^{23}\right), \\
& (\stackrel{3}{\varphi}, \stackrel{3}{\omega})_{e_{n}}=\sum_{k}\left(-\varphi_{k}^{012} \bar{\omega}_{k}^{012}-\varphi_{k}^{013} \bar{\omega}_{k}^{013}-\varphi_{k}^{023} \bar{\omega}_{k}^{023}+\varphi_{k}^{123} \bar{\omega}_{k}^{123}\right),
\end{aligned}
$$

where the sums are restricted to those sets of indices $k=\left(k_{0}, k_{1}, k_{2}, k_{3}\right)$ for which $1 \leq k_{\mu} \leq n_{\mu}$.

The remarkable feature here is that the $\cup$-product which has the property (2.15) is used in the definitions (2.24) and (2.16). This allows us to be more closely to continuum counterpart in the construction of a discrete codifferential as compared to the approach [17]. The following result is taken from [15, Proposition 2].

Proposition 2.2. Let $\stackrel{r}{\varphi} \in K^{r}(4)$ and $\stackrel{r+1}{\omega} \in K^{r+1}(4), r=0,1,2,3$. Then we have

$$
\left(d^{c} \stackrel{r}{\varphi}, \stackrel{r+1}{\omega}\right)_{e_{n}}=\left(\stackrel{r}{\varphi}, \delta^{c} \stackrel{r+1}{\omega}\right)_{e_{n}},
$$

where

$$
\delta^{c} \stackrel{r+1}{\omega}^{+1}(-1)^{r+1} *^{-1} d^{c} * \stackrel{r+1}{\omega}
$$

is the operator formally adjoint of $d^{c}$.

Here $*^{-1}$ is the inverse of $*$, i.e., $* *^{-1}=1$.

The operator $\delta^{c}: K^{r+1}(4) \rightarrow K^{r}(4)$ is a discrete analog of the codifferential $\delta$. For the 0 -form $\stackrel{0}{\omega} \in K^{0}(4)$ we have $\delta^{c} \stackrel{0}{\omega}=0$. It is obvious from (2.25) that $\delta^{c} \delta^{c} \stackrel{r}{\omega}=0$ for any $r=1,2,3,4$. Using (2.11)-(2.13) and (2.25) we can calculate

$$
\begin{aligned}
\delta^{c} \omega & =\sum_{k}\left(\Delta_{0}^{-} \omega_{k}^{0}-\Delta_{1}^{-} \omega_{k}^{1}-\Delta_{2}^{-} \omega_{k}^{2}-\Delta_{3}^{-} \omega_{k}^{3}\right) x^{k} \\
\delta^{c} \stackrel{\omega}{\omega}= & \sum_{k}\left[\left(\Delta_{1}^{-} \omega_{k}^{01}+\Delta_{2}^{-} \omega_{k}^{02}+\Delta_{3}^{-} \omega_{k}^{03}\right) e_{0}^{k}\right. \\
& +\left(\Delta_{0}^{-} \omega_{k}^{01}+\Delta_{2}^{-} \omega_{k}^{12}+\Delta_{3}^{-} \omega_{k}^{13}\right) e_{1}^{k} \\
& +\left(\Delta_{0}^{-} \omega_{k}^{02}-\Delta_{1}^{-} \omega_{k}^{12}+\Delta_{3}^{-} \omega_{k}^{23}\right) e_{2}^{k} \\
& \left.+\left(\Delta_{0}^{-} \omega_{k}^{03}-\Delta_{1}^{-} \omega_{k}^{13}-\Delta_{2}^{-} \omega_{k}^{23}\right) e_{3}^{k}\right]
\end{aligned}
$$




$$
\begin{aligned}
\delta^{c} \stackrel{3}{\omega}= & \sum_{k}\left[\left(-\Delta_{2}^{-} \omega_{k}^{012}-\Delta_{3}^{-} \omega_{k}^{013}\right) e_{01}^{k}+\left(\Delta_{1}^{-} \omega_{k}^{012}-\Delta_{3}^{-} \omega_{k}^{023}\right) e_{02}^{k}\right. \\
& +\left(\Delta_{1}^{-} \omega_{k}^{013}+\Delta_{2}^{-} \omega_{k}^{023}\right) e_{03}^{k}+\left(\Delta_{0}^{-} \omega_{k}^{012}-\Delta_{3}^{-} \omega_{k}^{123}\right) e_{12}^{k} \\
& \left.+\left(\Delta_{0}^{-} \omega_{k}^{013}+\Delta_{2}^{-} \omega_{k}^{123}\right) e_{13}^{k}+\left(\Delta_{0}^{-} \omega_{k}^{023}-\Delta_{1}^{-} \omega_{k}^{123}\right) e_{23}^{k}\right] \\
\delta^{c} \stackrel{4}{\omega}= & \sum_{k}\left[\left(\Delta_{3}^{-} \stackrel{4}{\omega}_{k}\right) e_{012}^{k}-\left(\Delta_{2}^{-} \stackrel{4}{\omega}_{k}\right) e_{013}^{k}+\left(\Delta_{1}^{-} \stackrel{4}{\omega}_{k}\right) e_{023}^{k}+\left(\Delta_{0}^{-} \stackrel{4}{\omega}_{k}\right) e_{123}^{k}\right] .
\end{aligned}
$$

Note that formulas (2.27)-(2.30) are essentially the same as the corresponding formulas from [15] up to notation.

As in the continuum case, the discrete codifferencial does not depend on the signature of the inner product, i.e., the operator $\delta^{c}$ is the same in both $(+---)$ and $(-+++)$ cases. Recall that in the continuum case we have $\delta=* d *$. However in the discrete case the operator $\delta^{c}$ is not equal to $* d^{c} *$. Indeed, let

$$
\delta^{c}{ }^{r+1}=\stackrel{r}{\omega}=\sum_{k} \sum_{(r)} \psi_{k}^{(r)} s_{(r)}^{k}, \quad r=0,1,2,3,
$$

but an easy computation shows that

$$
* d^{c} * \stackrel{r+1}{\omega}=\sum_{k} \sum_{(r)} \psi_{\sigma k}^{(r)} s_{(r)}^{k},
$$

where $\sigma k$ is given by $(2.22)$. Therefore in comparison with $\delta^{c^{r+1}} \omega$, the form $* d^{c} *{ }^{r+1}$ has the same components which are shifting to the left by one by all indexes. For example,

$$
* d^{c} * \stackrel{1}{\omega}=\sum_{k}\left(\Delta_{0}^{-} \omega_{\sigma k}^{0}-\Delta_{1}^{-} \omega_{\sigma k}^{1}-\Delta_{2}^{-} \omega_{\sigma k}^{2}-\Delta_{3}^{-} \omega_{\sigma k}^{3}\right) x^{k}
$$

while $\delta^{c} \stackrel{1}{\omega}$ has the view $(2.27)$.

\section{Discrete Dirac-Kähler and Joyce Equations}

In this section, we describe the difference representations of discrete DiracKähler and Joyce equations. We use the same technique as in [17]. However due to the definitions (2.16), (2.24) and (2.26) the discrete models here differ from ones in [17] and are more like their continuum counterparts. Availability of the difference operators both the forward and back type is an advantage of this approach. Let $\Omega$ be a discrete inhomogeneous form, that is

$$
\Omega=\sum_{r=0}^{4} \stackrel{r}{\omega}
$$

where $\stackrel{r}{\omega}$ is given by $(2.4)$. An alternative notation includes $(\Omega)_{r}=\stackrel{r}{\omega}$ for the $r$-form part of an inhomogeneous form. 
Introduce a discrete analog of the Dirac-Kähler equation (1.3) by the rule

$$
i\left(d^{c}+\delta^{c}\right) \Omega=m \Omega,
$$

where $m$ is a positive number (mass parameter). We can write this equation more explicitly by separating its homogeneous components as

$$
\begin{aligned}
i \delta^{c} \stackrel{1}{\omega} & =m \stackrel{0}{\omega}, \quad i\left(d^{c} \stackrel{1}{\omega}+\delta^{c} \stackrel{3}{\omega}\right)=m \stackrel{2}{\omega}, \quad i d^{c} \stackrel{3}{\omega}=m \stackrel{4}{\omega}, \\
i\left(d^{c} \stackrel{0}{\omega}+\delta^{c} \stackrel{2}{\omega}\right) & =m \stackrel{1}{\omega}, \quad i\left(d^{c} \stackrel{2}{\omega}+\delta^{c} \stackrel{4}{\omega}\right)=m \stackrel{3}{\omega} .
\end{aligned}
$$

Using (2.11)-(2.13) and (2.27)-(2.30) Eqs. (3.3) can be written as the set of following difference equations

$$
\begin{aligned}
i\left(\Delta_{0}^{-} \omega_{k}^{0}-\Delta_{1}^{-} \omega_{k}^{1}-\Delta_{2}^{-} \omega_{k}^{2}-\Delta_{3}^{-} \omega_{k}^{3}\right) & =m \omega_{k}^{0}, \\
i\left(\Delta_{0}^{+} \omega_{k}+\Delta_{1}^{-} \omega_{k}^{01}+\Delta_{2}^{-} \omega_{k}^{02}+\Delta_{3}^{-} \omega_{k}^{03}\right) & =m \omega_{k}^{0}, \\
i\left(\Delta_{1}^{+} \stackrel{\omega}{\omega}_{k}+\Delta_{0}^{-} \omega_{k}^{01}+\Delta_{2}^{-} \omega_{k}^{12}+\Delta_{3}^{-} \omega_{k}^{13}\right) & =m \omega_{k}^{1}, \\
i\left(\Delta_{2}^{+} \omega_{k}+\Delta_{0}^{-} \omega_{k}^{02}-\Delta_{1}^{-} \omega_{k}^{12}+\Delta_{3}^{-} \omega_{k}^{23}\right) & =m \omega_{k}^{2}, \\
i\left(\Delta_{3}^{+} \omega^{0}+\Delta_{0}^{-} \omega_{k}^{03}-\Delta_{1}^{-} \omega_{k}^{13}-\Delta_{2}^{-} \omega_{k}^{23}\right) & =m \omega_{k}^{3}, \\
i\left(\Delta_{0}^{+} \omega_{k}^{1}-\Delta_{1}^{+} \omega_{k}^{0}-\Delta_{2}^{-} \omega_{k}^{012}-\Delta_{3}^{-} \omega_{k}^{013}\right) & =m \omega_{k}^{01}, \\
i\left(\Delta_{0}^{+} \omega_{k}^{2}-\Delta_{2}^{+} \omega_{k}^{0}+\Delta_{1}^{-} \omega_{k}^{012}-\Delta_{3}^{-} \omega_{k}^{023}\right) & =m \omega_{k}^{02}, \\
i\left(\Delta_{0}^{+} \omega_{k}^{3}-\Delta_{3}^{+} \omega_{k}^{0}+\Delta_{1}^{-} \omega_{k}^{013}+\Delta_{2}^{-} \omega_{k}^{023}\right) & =m \omega_{k}^{03}, \\
i\left(\Delta_{1}^{+} \omega_{k}^{2}-\Delta_{2}^{+} \omega_{k}^{1}+\Delta_{0}^{-} \omega_{k}^{012}-\Delta_{3}^{-} \omega_{k}^{123}\right) & =m \omega_{k}^{12} \\
i\left(\Delta_{1}^{+} \omega_{k}^{3}-\Delta_{3}^{+} \omega_{k}^{1}+\Delta_{0}^{-} \omega_{k}^{013}+\Delta_{2}^{-} \omega_{k}^{123}\right) & =m \omega_{k}^{13}, \\
i\left(\Delta_{2}^{+} \omega_{k}^{3}-\Delta_{3}^{+} \omega_{k}^{2}+\Delta_{0}^{-} \omega_{k}^{023}-\Delta_{1}^{-} \omega_{k}^{123}\right) & =m \omega_{k}^{23} \\
i\left(\Delta_{0}^{+} \omega_{k}^{12}-\Delta_{1}^{+} \omega_{k}^{02}+\Delta_{2}^{+} \omega_{k}^{01}+\Delta_{3}^{-} \omega_{k}\right) & =m \omega_{k}^{012}, \\
i\left(\Delta_{0}^{+} \omega_{k}^{13}-\Delta_{1}^{+} \omega_{k}^{03}+\Delta_{3}^{+} \omega_{k}^{01}-\Delta_{2}^{-} \omega_{k}\right) & =m \omega_{k}^{013}, \\
i\left(\Delta_{0}^{+} \omega_{k}^{23}-\Delta_{2}^{+} \omega_{k}^{03}+\Delta_{3}^{+} \omega_{k}^{02}+\Delta_{1}^{-} \omega_{k}\right) & =m \omega_{k}^{023}, \\
i\left(\Delta_{1}^{+} \omega_{k}^{23}-\Delta_{2}^{+} \omega_{k}^{13}+\Delta_{3}^{+} \omega_{k}^{12}+\Delta_{0}^{-} \omega_{k}\right) & =m \omega_{k}^{123}, \\
i\left(\Delta_{0}^{+} \omega_{k}^{123}-\Delta_{1}^{+} \omega_{k}^{023}+\Delta_{2}^{+} \omega_{k}^{013}-\Delta_{3}^{+} \omega_{k}^{012}\right) & =m \omega_{k}
\end{aligned}
$$

Note that the equations above contain the difference operators both the forward and backward types. This is in contrast with the situation described in [15], where difference operators of the forward type only are used for the discrete construction.

For convenience, we recall the definition of the Clifford multiplication in $K(4)$ which has been previously used in $[17,19]$. The Clifford multiplication of the basis elements $x^{k}$ and $e_{\mu}^{k}, \mu=0,1,2,3$, is defined by
(a) $x^{k} x^{k}=x^{k}, \quad x^{k} e_{\mu}^{k}=e_{\mu}^{k} x^{k}=e_{\mu}^{k}$,
(b) $e_{\mu}^{k} e_{\nu}^{k}+e_{\nu}^{k} e_{\mu}^{k}=2 g_{\mu \nu} x^{k}, \quad g_{\mu \nu}=\operatorname{diag}(1,-1,-1,-1)$,
(c) $e_{\mu_{1}}^{k} \cdots e_{\mu_{s}}^{k}=e_{\mu_{1} \cdots \mu_{s}}^{k}$ for $\quad 0 \leq \mu_{1}<\cdots<\mu_{s} \leq 3$, 
supposing the product to be zero in all other cases. The operation is linearly extended to arbitrary discrete forms.

Consider the following unit forms

$$
x=\sum_{k} x^{k}, \quad e=\sum_{k} e^{k}, \quad e_{\mu}=\sum_{k} e_{\mu}^{k}, \quad e_{\mu \nu}=\sum_{k} e_{\mu \nu}^{k} .
$$

Note that the unit 0 -form $x$ plays a role of the unit element in $K(4)$ with respect to the Clifford multiplication, i.e., for $\stackrel{r}{\omega} \in K^{r}(4)$ we get $x \stackrel{r}{\omega}=\stackrel{r}{\omega} x=\stackrel{r}{\omega}$. It is evident that

$$
e_{\mu} e_{\nu}+e_{\nu} e_{\mu}=2 g_{\mu \nu} x, \quad \mu, \nu=0,1,2,3 .
$$

Proposition 3.1. For any inhomogeneous form $\Omega \in K(4)$ we have

$$
\left(d^{c}+\delta^{c}\right) \Omega=\sum_{r=0}^{3}\left(\sum_{\mu=0}^{3} e_{\mu} \Delta_{\mu}^{+} \stackrel{r}{\omega}\right)_{r+1}+\sum_{r=1}^{4}\left(\sum_{\mu=0}^{3} e_{\mu} \Delta_{\mu}^{-} \stackrel{r}{\omega}\right)_{r-1},
$$

where $\Delta_{\mu}^{+}$and $\Delta_{\mu}^{-}$are the difference operators which act on each component of $\stackrel{r}{\omega}$ by the rules $(2.8)$ and $(2.9)$.

Proof. By (2.11), it is clear that

$$
d^{c} \stackrel{0}{\omega}=\sum_{\mu=0}^{3} e_{\mu} \Delta_{\mu}^{+} \stackrel{0}{\omega}=\left(\sum_{\mu=0}^{3} e_{\mu} \Delta_{\mu}^{+} \stackrel{0}{\omega}\right)_{1}
$$

In the case of the 2 -form $\stackrel{2}{\omega}$ we compute

$$
\begin{aligned}
\sum_{\mu=0}^{3} e_{\mu} \Delta_{\mu}^{ \pm} \omega= & \left(\sum_{\mu=0}^{3} e_{\mu} \Delta_{\mu}^{ \pm} \omega\right)_{1}+\left(\sum_{\mu=0}^{3} e_{\mu} \Delta_{\mu}^{ \pm} \omega\right)_{3} \\
= & \sum_{k}\left[\left(\Delta_{1}^{ \pm} \omega_{k}^{01}+\Delta_{2}^{ \pm} \omega_{k}^{02}+\Delta_{3}^{ \pm} \omega_{k}^{03}\right) e_{0}^{k}\right. \\
& +\left(\Delta_{0}^{ \pm} \omega_{k}^{01}+\Delta_{2}^{ \pm} \omega_{k}^{12}+\Delta_{3}^{ \pm} \omega_{k}^{13}\right) e_{1}^{k} \\
& +\left(\Delta_{0}^{ \pm} \omega_{k}^{02}-\Delta_{1}^{ \pm} \omega_{k}^{12}+\Delta_{3}^{ \pm} \omega_{k}^{23}\right) e_{2}^{k} \\
& \left.+\left(\Delta_{0}^{ \pm} \omega_{k}^{03}-\Delta_{1}^{ \pm} \omega_{k}^{13}-\Delta_{2}^{ \pm} \omega_{k}^{23}\right) e_{3}^{k}\right] \\
& +\sum_{k}\left[\left(\Delta_{0}^{ \pm} \omega_{k}^{12}-\Delta_{1}^{ \pm} \omega_{k}^{02}+\Delta_{2}^{ \pm} \omega_{k}^{01}\right) e_{012}^{k}\right. \\
& +\left(\Delta_{0}^{ \pm} \omega_{k}^{13}-\Delta_{1}^{ \pm} \omega_{k}^{03}+\Delta_{3}^{ \pm} \omega_{k}^{01}\right) e_{013}^{k} \\
& +\left(\Delta_{0}^{ \pm} \omega_{k}^{23}-\Delta_{2}^{ \pm} \omega_{k}^{03}+\Delta_{3}^{ \pm} \omega_{k}^{02}\right) e_{023}^{k} \\
& \left.+\left(\Delta_{1}^{ \pm} \omega_{k}^{23}-\Delta_{2}^{ \pm} \omega_{k}^{13}+\Delta_{3}^{ \pm} \omega_{k}^{12}\right) e_{123}^{k}\right] .
\end{aligned}
$$

From this using (2.12) and (2.28) we have

$$
d^{c} \stackrel{2}{\omega}=\left(\sum_{\mu=0}^{3} e_{\mu} \Delta_{\mu}^{+} \stackrel{2}{\omega}\right)_{3}
$$


and

$$
\delta^{c} \stackrel{2}{\omega}=\left(\sum_{\mu=0}^{3} e_{\mu} \Delta_{\mu}^{-2} \stackrel{2}{\omega}\right)_{1}
$$

For $\stackrel{4}{\omega}$ we obtain

$$
\sum_{\mu=0}^{3} e_{\mu} \Delta_{\mu}^{-} \stackrel{4}{\omega}=\sum_{k}\left(\Delta_{0}^{-} \stackrel{4}{\omega}_{k} e_{123}^{k}+\Delta_{1}^{-} \stackrel{4}{\omega}_{k} e_{023}^{k}-\Delta_{2}^{-} \stackrel{4}{\omega}_{k} e_{013}^{k}+\Delta_{3}^{-} \stackrel{4}{\omega}_{k} e_{012}^{k}\right)
$$

By (2.30), we have

$$
\delta^{c} \stackrel{4}{\omega}=\sum_{\mu=0}^{3} e_{\mu} \Delta_{\mu}^{-} \stackrel{4}{\omega}=\left(\sum_{\mu=0}^{3} e_{\mu} \Delta_{\mu}^{-} \stackrel{4}{\omega}\right)_{3}
$$

Similar calculations apply to the case of odd forms. Hence

$$
d^{c} \stackrel{r}{\omega}=\left(\sum_{\mu=0}^{3} e_{\mu} \Delta_{\mu}^{+} \stackrel{r}{\omega}\right)_{r+1} \text { for } \quad r=0,1,2,3
$$

and

$$
\delta^{c} \stackrel{r}{\omega}=\left(\sum_{\mu=0}^{3} e_{\mu} \Delta_{\mu}^{-} \stackrel{r}{\omega}\right)_{r-1} \quad \text { for } \quad r=1,2,3,4 .
$$

Combining (3.7) and (3.8) yields (3.6).

Clearly, the discrete Dirac-Kähler equation (3.2) can be rewritten in the form

$$
i\left(\sum_{r=0}^{3}\left(\sum_{\mu=0}^{3} e_{\mu} \Delta_{\mu}^{+} \stackrel{r}{\omega}\right)_{r+1}+\sum_{r=1}^{4}\left(\sum_{\mu=0}^{3} e_{\mu} \Delta_{\mu}^{-} \stackrel{r}{\omega}\right)_{r-1}\right)=m \sum_{r=0}^{4} \stackrel{r}{\omega}
$$

Let $K^{e v}(4)=K^{0}(4) \oplus K^{2}(4) \oplus K^{4}(4)$ and let $\Omega^{e v} \in K^{e v}(4)$ be a complexvalued even inhomogeneous form, i.e., $\Omega^{e v}=\stackrel{0}{\omega}+\stackrel{2}{\omega}+\stackrel{4}{\omega}$. A discrete analogue of the Joyce equation (1.8) is defined by

$$
i\left(d^{c}+\delta^{c}\right) \Omega^{e v}=m \Omega^{e v} e_{0}
$$

where $e_{0}$ is given by (3.4). From (3.6) it follows that Eq. (3.9) is equivalent to

$$
i\left(\sum_{\mu=0}^{3} e_{\mu} \Delta_{\mu}^{+} \stackrel{0}{\omega}+\left(\sum_{\mu=0}^{3} e_{\mu} \Delta_{\mu}^{+} \stackrel{2}{\omega}\right)_{3}+\left(\sum_{\mu=0}^{3} e_{\mu} \Delta_{\mu}^{-2} \omega\right)_{1}+\sum_{\mu=0}^{3} e_{\mu} \Delta_{\mu}^{-} \stackrel{4}{\omega}\right)=m \Omega^{e v} e_{0} .
$$

A more detailed calculation leads to the following system of 8 difference equations

$$
\begin{aligned}
& i\left(\Delta_{0}^{+} \stackrel{0}{\omega}_{k}+\Delta_{1}^{-} \omega_{k}^{01}+\Delta_{2}^{-} \omega_{k}^{02}+\Delta_{3}^{-} \omega_{k}^{03}\right)=m \omega^{0}, \\
& i\left(\Delta_{1}^{+} \stackrel{0}{\omega}_{k}+\Delta_{0}^{-} \omega_{k}^{01}+\Delta_{2}^{-} \omega_{k}^{12}+\Delta_{3}^{-} \omega_{k}^{13}\right)=-m \omega_{k}^{01}, \\
& i\left(\Delta_{2}^{+} \stackrel{0}{\omega}_{k}+\Delta_{0}^{-} \omega_{k}^{02}-\Delta_{1}^{-} \omega_{k}^{12}+\Delta_{3}^{-} \omega_{k}^{23}\right)=-m \omega_{k}^{02}, \\
& i\left(\Delta_{3}^{+} \stackrel{0}{\omega}_{k}+\Delta_{0}^{-} \omega_{k}^{03}-\Delta_{1}^{-} \omega_{k}^{13}-\Delta_{2}^{-} \omega_{k}^{23}\right)=-m \omega_{k}^{03},
\end{aligned}
$$




$$
\begin{aligned}
& i\left(\Delta_{0}^{+} \omega_{k}^{12}-\Delta_{1}^{+} \omega_{k}^{02}+\Delta_{2}^{+} \omega_{k}^{01}+\Delta_{3}^{-} \stackrel{4}{\omega}_{k}\right)=m \omega_{k}^{12}, \\
& i\left(\Delta_{0}^{+} \omega_{k}^{13}-\Delta_{1}^{+} \omega_{k}^{03}+\Delta_{3}^{+} \omega_{k}^{01}-\Delta_{2}^{-} \stackrel{4}{\omega}_{k}\right)=m \omega_{k}^{13}, \\
& i\left(\Delta_{0}^{+} \omega_{k}^{23}-\Delta_{2}^{+} \omega_{k}^{03}+\Delta_{3}^{+} \omega_{k}^{02}+\Delta_{1}^{-} \stackrel{4}{\omega}_{k}\right)=m \omega_{k}^{23}, \\
& i\left(\Delta_{1}^{+} \omega_{k}^{23}-\Delta_{2}^{+} \omega_{k}^{13}+\Delta_{3}^{+} \omega_{k}^{12}+\Delta_{0}^{-} \stackrel{4}{\omega}_{k}\right)=-m \stackrel{4}{\omega}_{k}
\end{aligned}
$$

for each $k=\left(k_{0}, k_{1}, k_{2}, k_{3}\right)$.

\section{Plane Wave Solutions}

In this section, we consider solutions of the discrete Joyce equation which imitate the plane wave solutions for the continuum counterpart. We will mainly follow the strategy of [18], but there are extra technical difficulties here. These difficulties arise in the construction of eigenvectors of the operator $i\left(d^{c}+\delta^{c}\right)$ since now this operator consists of difference operators of the two types. It is necessary to slightly modify the construction of a solution to obtain results similar to the case of the discrete Hestenes equation in the approach [17].

Let us consider the complex-valued 0 -forms

$$
\psi^{0}=\sum_{k} \psi_{k}^{0} x^{k}, \quad \psi^{\mu \nu}=\sum_{k} \psi_{k}^{\mu \nu} x^{k}, \quad \psi^{4}=\sum_{k} \psi_{k}^{4} x^{k},
$$

where

$$
\begin{aligned}
\psi_{k}^{0} & =\left(1+i p_{0}\right)^{k_{0}}\left(1+i p_{1}\right)^{k_{1}}\left(1+i p_{2}\right)^{k_{2}}\left(1+i p_{3}\right)^{k_{3}}, \\
\psi_{k}^{01} & =\left(1-i p_{0}\right)^{-k_{0}}\left(1-i p_{1}\right)^{-k_{1}}\left(1+i p_{2}\right)^{k_{2}}\left(1+i p_{3}\right)^{k_{3}}, \\
\psi_{k}^{02} & =\left(1-i p_{0}\right)^{-k_{0}}\left(1+i p_{1}\right)^{k_{1}}\left(1-i p_{2}\right)^{-k_{2}}\left(1+i p_{3}\right)^{k_{3}}, \\
\psi_{k}^{03} & =\left(1-i p_{0}\right)^{-k_{0}}\left(1+i p_{1}\right)^{k_{1}}\left(1+i p_{2}\right)^{k_{2}}\left(1-i p_{3}\right)^{-k_{3}}, \\
\psi_{k}^{12} & =\left(1+i p_{0}\right)^{k_{0}}\left(1-i p_{1}\right)^{-k_{1}}\left(1-i p_{2}\right)^{-k_{2}}\left(1+i p_{3}\right)^{k_{3}}, \\
\psi_{k}^{13} & =\left(1+i p_{0}\right)^{k_{0}}\left(1-i p_{1}\right)^{-k_{1}}\left(1+i p_{2}\right)^{k_{2}}\left(1-i p_{3}\right)^{-k_{3}}, \\
\psi_{k}^{23} & =\left(1+i p_{0}\right)^{k_{0}}\left(1+i p_{1}\right)^{k_{1}}\left(1-i p_{2}\right)^{-k_{2}}\left(1-i p_{3}\right)^{-k_{3}}, \\
\psi_{k}^{4} & =\left(1-i p_{0}\right)^{-k_{0}}\left(1-i p_{1}\right)^{-k_{1}}\left(1-i p_{2}\right)^{-k_{2}}\left(1-i p_{3}\right)^{-k_{3}},
\end{aligned}
$$

and $p_{\mu} \in \mathbb{R}$. It is easy to check that

$$
\begin{aligned}
\Delta_{\mu}^{+}\left(\left(1+i p_{\mu}\right)^{k_{\mu}}\right) & =\left(1+i p_{\mu}\right)^{k_{\mu}+1}-\left(1+i p_{\mu}\right)^{k_{\mu}}=i p_{\mu}\left(1+i p_{\mu}\right)^{k_{\mu}}, \\
\Delta_{\mu}^{-}\left(\left(1-i p_{\mu}\right)^{-k_{\mu}}\right) & =\left(1-i p_{\mu}\right)^{-k_{\mu}}-\left(1-i p_{\mu}\right)^{-\left(k_{\mu}-1\right)}=i p_{\mu}\left(1-i p_{\mu}\right)^{-k_{\mu}} .
\end{aligned}
$$

As a consequence we obtain

$$
\begin{aligned}
\Delta_{\mu}^{+} \psi_{k}^{0} & =i p_{\mu} \psi_{k}^{0}, \quad \Delta_{\mu}^{-} \psi_{k}^{4}=i p_{\mu} \psi_{k}^{4}, \\
\Delta_{\mu}^{-} \psi_{k}^{\mu \nu} & =i p_{\mu} \psi_{k}^{\mu \nu}, \quad \Delta_{\nu}^{-} \psi_{k}^{\mu \nu}=i p_{\nu} \psi_{k}^{\mu \nu}, \\
\Delta_{\iota}^{+} \psi_{k}^{\mu \nu} & =i p_{\iota} \psi_{k}^{\mu \nu}, \quad \text { for } \quad \iota \neq \mu, \nu .
\end{aligned}
$$


Let $A \in K^{e v}(4)$ is a constant complex-valued form. Hence $A$ can be expanded as

$$
A=\alpha^{0} x+\sum_{\mu<\nu} \alpha^{\mu \nu} e_{\mu \nu}+\alpha^{4} e
$$

where $\alpha^{0}, \alpha^{\mu \nu}, \alpha^{4} \in \mathbb{C}$ and $x, e_{\mu \nu}, e$ are the unit forms given by (3.4). Consider the form

$$
\Phi=\stackrel{0}{\varphi}+\stackrel{2}{\varphi}+\stackrel{4}{\varphi}
$$

where

$$
\stackrel{0}{\varphi}=\alpha^{0} \psi^{0}, \quad \stackrel{2}{\varphi}=\sum_{\mu<\nu} \alpha^{\mu \nu} \psi^{\mu \nu} e_{\mu \nu}, \quad \stackrel{4}{\varphi}=\alpha^{4} \psi^{4} e,
$$

and $\psi^{0}, \psi^{\mu \nu}$ and $\psi^{4}$ are given by (4.1).

Proposition 4.1. For the form (4.6) we have that

$$
\left(d^{c}+\delta^{c}\right) \Phi=i\left(\sum_{\mu=0}^{3} e_{\mu} p_{\mu}\right) \Phi .
$$

Proof. Apply the operators $d^{c}$ and $\delta^{c}$ to the forms $\stackrel{0}{\varphi}, \stackrel{2}{\varphi}$ and $\stackrel{4}{\varphi}$. The calculations are similar to the ones in the proof of Proposition 3.1. Using (3.7), (3.8) and (4.2) we obtain

$$
d^{c} \stackrel{0}{\varphi}=\sum_{\mu=0}^{3} e_{\mu} \Delta_{\mu}^{+} \stackrel{0}{\varphi}=\sum_{\mu=0}^{3} e_{\mu} \alpha^{0} \sum_{k}\left(\Delta_{\mu}^{+} \psi_{k}^{0}\right) x^{k}=i\left(\sum_{\mu=0}^{3} e_{\mu} p_{\mu}\right)^{0}
$$

and

$$
\delta^{c} \stackrel{4}{\varphi}=\sum_{\mu=0}^{3} e_{\mu} \Delta_{\mu}^{-} \stackrel{4}{\varphi}=\sum_{\mu=0}^{3} e_{\mu} \alpha^{4} \sum_{k}\left(\Delta_{\mu}^{-} \psi_{k}^{4}\right) x^{k} e=i\left(\sum_{\mu=0}^{3} e_{\mu} p_{\mu}\right) \stackrel{4}{\varphi}
$$

By (4.3) and (4.4), we calculate

$$
\begin{aligned}
d^{c} \stackrel{\varphi}{\varphi}= & \left(\sum_{\mu=0}^{3} e_{\mu} \Delta_{\mu}^{+} \stackrel{2}{\varphi}\right)_{3}=i p_{0} e_{0}\left(\alpha^{12} \psi^{12} e_{12}+\alpha^{13} \psi^{13} e_{13}+\alpha^{23} \psi^{23} e_{23}\right) \\
& +i p_{1} e_{1}\left(\alpha^{02} \psi^{02} e_{02}+\alpha^{03} \psi^{03} e_{03}+\alpha^{23} \psi^{23} e_{23}\right) \\
& +i p_{2} e_{2}\left(\alpha^{01} \psi^{01} e_{01}+\alpha^{03} \psi^{03} e_{03}+\alpha^{13} \psi^{13} e_{13}\right) \\
& +i p_{3} e_{3}\left(\alpha^{01} \psi^{01} e_{01}+\alpha^{02} \psi^{02} e_{02}+\alpha^{12} \psi^{12} e_{12}\right)
\end{aligned}
$$

and

$$
\begin{aligned}
\delta^{c} \stackrel{\varphi}{\varphi}= & \left(\sum_{\mu=0}^{3} e_{\mu} \Delta_{\mu}^{-2}\right)_{1}=i p_{0} e_{0}\left(\alpha^{01} \psi^{01} e_{01}+\alpha^{02} \psi^{02} e_{02}+\alpha^{03} \psi^{03} e_{03}\right) \\
& +i p_{1} e_{1}\left(\alpha^{01} \psi^{01} e_{01}+\alpha^{12} \psi^{12} e_{12}+\alpha^{13} \psi^{13} e_{13}\right) \\
& +i p_{2} e_{2}\left(\alpha^{12} \psi^{12} e_{12}+\alpha^{02} \psi^{02} e_{02}+\alpha^{23} \psi^{23} e_{23}\right) \\
& +i p_{3} e_{3}\left(\alpha^{03} \psi^{03} e_{03}+\alpha^{13} \psi^{13} e_{13}+\alpha^{23} \psi^{23} e_{23}\right)
\end{aligned}
$$


This yields

$$
\left(d^{c}+\delta^{c}\right)^{2} \varphi=i\left(\sum_{\mu=0}^{3} e_{\mu} p_{\mu}\right)^{2} \varphi
$$

Summing up we get (4.7).

Now we seek the general solution to the discrete Joyce equation which we assume to be of the form (4.6). Substituting (4.7) into Eq. (3.9) we obtain

$$
-\left(\sum_{\mu=0}^{3} e_{\mu} p_{\mu}\right) \Phi=m \Phi e_{0} .
$$

It is not difficult to show that Eq. (4.8) is equivalent to the following system of equations

$$
\begin{aligned}
& \left(p_{0}+m\right) \alpha^{0} \psi^{0}+p_{1} \alpha^{01} \psi^{01}+p_{2} \alpha^{02} \psi^{02}+p_{3} \alpha^{03} \psi^{03}=0 \\
& \left(p_{0}+m\right) \alpha^{12} \psi^{12}-p_{1} \alpha^{02} \psi^{02}+p_{2} \alpha^{01} \psi^{01}-p_{3} \alpha^{4} \psi^{4}=0 \\
& \left(p_{0}+m\right) \alpha^{13} \psi^{13}-p_{1} \alpha^{03} \psi^{03}-p_{2} \alpha^{4} \psi^{4}+p_{3} \alpha^{01} \psi^{01}=0 \\
& \left(p_{0}+m\right) \alpha^{23} \psi^{23}+p_{1} \alpha^{4} \psi^{4}-p_{2} \alpha^{03} \psi^{03}+p_{3} \alpha^{02} \psi^{02}=0 \\
& \left(p_{0}-m\right) \alpha^{01} \psi^{01}+p_{1} \alpha^{0} \psi^{0}+p_{2} \alpha^{12} \psi^{12}+p_{3} \alpha^{13} \psi^{13}=0 \\
& \left(p_{0}-m\right) \alpha^{02} \psi^{02}-p_{1} \alpha^{12} \psi^{12}+p_{2} \alpha^{0} \psi^{0}+p_{3} \alpha^{23} \psi^{23}=0 \\
& \left(p_{0}-m\right) \alpha^{03} \psi^{03}-p_{1} \alpha^{13} \psi^{13}-p_{2} \alpha^{23} \psi^{23}+p_{3} \alpha^{0} \psi^{0}=0 \\
& \left(p_{0}-m\right) \alpha^{4} \psi^{4}+p_{1} \alpha^{23} \psi^{23}-p_{2} \alpha^{13} \psi^{13}+p_{3} \alpha^{12} \psi^{12}=0 .
\end{aligned}
$$

Proposition 4.2. The form (4.6) is a non-trivial solution of Eq. (3.9) if and only if

$$
p_{0}^{2}=m^{2}+p_{1}^{2}+p_{2}^{2}+p_{3}^{2} .
$$

Proof. Multiplying from the left Eq. (4.8) by $e_{0}$ and keeping in mind that $e_{0} e_{0}=x$ we obtain

$$
-\left(p_{0} x+\sum_{\mu=1}^{3} p_{\mu} e_{0} e_{\mu}\right) \Phi=m e_{0} \Phi e_{0} .
$$

A direct computation shows that

$$
\left(p_{0} x-\sum_{\mu=1}^{3} p_{\mu} e_{0} e_{\mu}\right)\left(p_{0} x+\sum_{\mu=1}^{3} p_{\mu} e_{0} e_{\mu}\right)=\left(p_{0}^{2}-\sum_{\mu=1}^{3} p_{\mu}^{2}\right) x .
$$

Then multiplying both sides of Eq. (4.18) by the same factor: $-\left(p_{0} x-\right.$ $\left.\sum_{\mu=1}^{3} p_{\mu} e_{0} e_{\mu}\right)$ gives

$$
\left(p_{0}^{2}-\sum_{\mu=1}^{3} p_{\mu}^{2}\right) x \Phi=-m\left(p_{0} x-\sum_{\mu=1}^{3} p_{\mu} e_{0} e_{\mu}\right) e_{0} \Phi e_{0} .
$$

By (3.5), this gives the equation

$$
\left(p_{0}^{2}-\sum_{\mu=1}^{3} p_{\mu}^{2}\right) \Phi=-m\left(\sum_{\mu=0}^{3} p_{\mu} e_{\mu}\right) \Phi e_{0} .
$$


Applying (4.8) to the right-hand side we obtain

$$
\left(p_{0}^{2}-\sum_{\mu=1}^{3} p_{\mu}^{2}\right) \Phi=m^{2} \Phi e_{0} e_{0},
$$

or equivalently,

$$
\left(p_{0}^{2}-\sum_{\mu=1}^{3} p_{\mu}^{2}-m^{2}\right) \Phi=0 .
$$

This equation has non-trivial solutions if and only if $p_{0}^{2}-\sum_{\mu=1}^{3} p_{\mu}^{2}=m^{2}$.

Therefore, if we define $p=\left\{p_{0}, p_{1}, p_{2}, p_{3}\right\}$ to be the energy-momentum vector of a particle with (proper) mass $m$, then the relation (4.17) is the energy-momentum relation. In view of this, it makes sense to state that the form (4.6) is a discrete version of the plane wave solution. Recall that the Joyce equation (1.6) admits the plane wave solutions of the form (1.7). Thus the 0 -form $\psi=\psi^{0}+\psi^{\mu \nu}+\psi^{4}$ given by (4.1) plays a role of the function $\exp (i p \cdot x)$ in the discrete case.

It should be noted that the same proposition for a discrete analog of the Hestenes equation in the case of a discrete model based on the double complex construction is proven in [17].

In order to have an explicit formula for the general plane wave solution of Eq. (3.9) we decompose the form $\Phi$ into its commute and anticommute parts with respect to $e_{0}$. Our calculations here follow the same lines as in [17] for the discrete Hestenes equation. Let us represent the even complex-valued form (4.5) as

$$
A=A_{+}+A_{-},
$$

where

$$
\begin{aligned}
& A_{+}=\alpha^{0} x+\alpha^{12} e_{12}+\alpha^{13} e_{13}+\alpha^{23} e_{23}, \\
& A_{-}=\alpha^{01} e_{01}+\alpha^{02} e_{02}+\alpha^{03} e_{03}+\alpha^{4} e .
\end{aligned}
$$

It is easy to check that $A_{+}$commutes with $e_{0}$ and $A_{-}$anticommutes with it, i.e.,

$$
e_{0} A_{ \pm}= \pm A_{ \pm} e_{0} .
$$

Then the form (4.6) can be represented also as

$$
\Phi=\Phi_{+}+\Phi_{-},
$$

where

$$
\begin{aligned}
& \Phi_{+}=\alpha^{0} \psi^{0} x+\alpha^{12} \psi^{12} e_{12}+\alpha^{13} \psi^{13} e_{13}+\alpha^{23} \psi^{23} e_{23}, \\
& \Phi_{-}=\alpha^{01} \psi^{01} e_{01}+\alpha^{02} \psi^{02} e_{02}+\alpha^{03} \psi^{03} e_{03}+\alpha^{4} \psi^{4} e .
\end{aligned}
$$

Since the 0-forms (4.1) commute with $e_{0}$ then Eq. (4.19) is true also for $\Phi_{ \pm}$, i.e.,

$$
e_{0} \Phi_{ \pm}= \pm \Phi_{ \pm} e_{0}
$$

As is shown in [18], the proof of the following lemma follows from a direct computation. 
Lemma 4.3. The form $e_{0 \mu} \Phi_{-}$commutes with $e_{0}$ and has the view (4.20), while $e_{0 \mu} \Phi_{+}$anticommutes with $e_{0}$ and has the view (4.21) for any $\mu=1,2,3$.

Theorem 4.4. The form $\Phi$ given by (4.6) is a non-trivial solution of the discrete Joyce equation if and only if the condition

$$
\Phi_{-}=\frac{p_{1} e_{01}+p_{2} e_{02}+p_{3} e_{03}}{m-p_{0}} \Phi_{+}
$$

holds, or equivalently,

$$
\Phi_{+}=-\frac{p_{1} e_{01}+p_{2} e_{02}+p_{3} e_{03}}{m+p_{0}} \Phi_{-} .
$$

Proof. By Lemma 4.3 and (4.8), the proof coincides with the one contained in $[18$, Theorem 1].

It is not difficult to show that the condition (4.22) is equivalent to the system of Eqs. (4.13)-(4.16). Similarly, the condition (4.23) gives the system of Eqs. (4.9)-(4.12). Moreover, using Eqs. (4.13)-(4.16) the form (4.22) can be written as

$$
\begin{aligned}
\Phi_{-}= & \frac{p_{1} \alpha^{0} \psi^{0}+p_{2} \alpha^{12} \psi^{12}+p_{3} \alpha^{13} \psi^{13}}{m-p_{0}} e_{01} \\
& +\frac{p_{2} \alpha^{0} \psi^{0}-p_{1} \alpha^{12} \psi^{12}+p_{3} \alpha^{23} \psi^{23}}{m-p_{0}} e_{02} \\
& +\frac{p_{3} \alpha^{0} \psi^{0}-p_{1} \alpha^{13} \psi^{13}-p_{2} \alpha^{23} \psi^{23}}{m-p_{0}} e_{03} \\
& +\frac{p_{3} \alpha^{12} \psi^{12}-p_{2} \alpha^{13} \psi^{13}+p_{1} \alpha^{23} \psi^{23}}{m-p_{0}} e .
\end{aligned}
$$

Hence

$$
\begin{aligned}
\Phi= & a_{1} \psi^{0}\left(\left(m-p_{0}\right) x+p_{1} e_{01}+p_{2} e_{02}+p_{3} e_{03}\right) \\
& +a_{2} \psi^{12}\left(\left(m-p_{0}\right) e_{12}+p_{2} e_{01}-p_{1} e_{02}+p_{3} e\right) \\
& +a_{3} \psi^{13}\left(\left(m-p_{0}\right) e_{13}+p_{3} e_{01}-p_{1} e_{03}+p_{2} e\right) \\
& +a_{4} \psi^{23}\left(\left(m-p_{0}\right) e_{23}+p_{3} e_{02}-p_{2} e_{03}+p_{1} e\right),
\end{aligned}
$$

where

$$
a_{1}=\frac{\alpha^{0}}{m-p_{0}}, \quad a_{2}=\frac{\alpha^{12}}{m-p_{0}}, \quad a_{3}=\frac{\alpha^{13}}{m-p_{0}}, \quad a_{4}=\frac{\alpha^{23}}{m-p_{0}} .
$$

It turns out that the discrete general plane wave solution of Eq. (3.9) consists of four linearly independent solutions for given $p_{\mu}, \mu=1,2,3$. To be more precise, there are four linearly independent solutions for each positive and negative $p_{0}= \pm \sqrt{m^{2}+p_{1}^{2}+p_{2}^{2}+p_{3}^{2}}$. Thus the result is the same as for the continuum counterpart. The presence of eight such solutions in the continuum case is interpreted in details in [10].

Similarly, again, if we take the condition (4.23) and use Eqs. (4.9)-(4.12) then we obtain 


$$
\begin{aligned}
\Phi_{+}= & \frac{-p_{1} \alpha^{01} \psi^{01}-p_{2} \alpha^{02} \psi^{02}-p_{3} \alpha^{03} \psi^{03}}{m+p_{0}} x \\
& +\frac{p_{1} \alpha^{02} \psi^{02}-p_{2} \alpha^{01} \psi^{01}-p_{3} \alpha^{4} \psi^{4}}{m+p_{0}} e_{12} \\
& +\frac{p_{1} \alpha^{03} \psi^{03}+p_{2} \alpha^{4} \psi^{4}-p_{3} \alpha^{01} \psi^{01}}{m+p_{0}} e_{13} \\
& +\frac{-p_{1} \alpha^{4} \psi^{4}+p_{2} \alpha^{03} \psi^{03}-p_{3} \alpha^{02} \psi^{02}}{m+p_{0}} e_{23} .
\end{aligned}
$$

This leads to the general solution

$$
\begin{aligned}
\Phi= & b_{1} \psi^{01}\left(\left(m+p_{0}\right) e_{01}-p_{1} x-p_{2} e_{12}-p_{3} e_{13}\right) \\
& +b_{2} \psi^{02}\left(\left(m+p_{0}\right) e_{02}-p_{2} x+p_{1} e_{12}-p_{3} e_{23}\right) \\
& +b_{3} \psi^{03}\left(\left(m+p_{0}\right) e_{03}-p_{3} x+p_{1} e_{13}+p_{2} e_{23}\right) \\
& +b_{4} \psi^{4}\left(\left(m+p_{0}\right) e-p_{3} e_{12}+p_{2} e_{13}-p_{1} e_{23}\right),
\end{aligned}
$$

where

$$
b_{1}=\frac{\alpha^{01}}{m+p_{0}}, \quad b_{2}=\frac{\alpha^{02}}{m+p_{0}}, \quad b_{3}=\frac{\alpha^{03}}{m+p_{0}}, \quad b_{4}=\frac{\alpha^{4}}{m+p_{0}} .
$$

It is clear that the equivalence of (4.22) and (4.23) implies the equivalence of the solutions (4.24) and (4.25).

Remark 4.5. It should be noted that in the approach using in [17] a discrete plane wave solution of the discrete Joyce equation can be obtaned by taking the complex unit $i$ instead of $e_{12}$ in the construction of the 0 -form $\Psi^{ \pm}$(see $\left[17\right.$, formula (4.2)]). In the present approach it is not true. If we take $e_{12}$ instead $i$ in the forms (4.1) then the form $\Phi$ does not satisfy the relation (4.7). As a consequence of this we can not obtain a plane wave solution of the form (4.6) for the discrete Hestenes equation.

Remark 4.6. It is easy to show that if $\Omega \in K^{e v}(4)$ is a solution of the discrete Joyce equation (3.9) then the complex-valued form $\Omega P_{+12} \in K^{e v}(4)$, where $P_{+12}=\frac{1}{2}\left(x+i e_{1} e_{2}\right)$ is a projector such that $P_{+12}=i P_{+12} e_{1} e_{2}$, satisfies the discrete Hestenes equation. Moreover in [19], we proved that every solution of the discrete Joyce equation can be represented in the form

$$
\Omega=\frac{1}{2}\left(\Omega_{+}+\Omega_{-}\right)+\frac{i}{2}\left(\Omega_{+}-\Omega_{-}\right) e_{1} e_{2},
$$

where the real-valued even forms

$$
\Omega_{ \pm}=\frac{1}{2}(\Omega+\bar{\Omega}) \pm \frac{i}{2}(\Omega-\bar{\Omega}) e_{1} e_{2}
$$

are solutions of the discrete Hestenes equations with correct or reversed sign on the right hand side [19, Proposition 6]. Note that this result is true for both the double complex and the present approaches. Thus putting $\Omega=\Phi$ in (4.26), where $\Phi$ is the plane wave solution (4.24) (or (4.25)) we obtain a solution of the same type for the discrete Hestenes equation. 
Remark 4.\%. The discrete model admits the chiral symmetries and the chiral properties are captured for the discrete Joyce equation. By using the chiral projectors $P_{R}=\frac{1}{2}(x+i e)$ and $P_{L}=\frac{1}{2}(x-i e)$, where $x$ and $e$ are the unit 0 - and 4 -forms (3.4), any solution of the discrete Joyce equation (3.9) decomposes into its chiral right and chiral left parts. The chirality for discrete plane wave solutions is the subject of current work in progress.

\section{Conclusions}

In this work, a discrete version of the plane wave solution to the discrete Dirac-Kähler equation in the Joyce form is constructed. We have shown that this discrete model preserves the key properties of the continuum counterpart. The proposed geometric discretisation scheme makes it possible to define discrete analogs of the differential and codifferential by using both forward and backward difference operators. Thus in comparison with an approach based on the double complex construction [17,18], we have obtained a discrete analog more suitable for applied purposes.

\section{Acknowledgements}

The author is grateful to the referees for careful reviews and fruitful suggestions.

Open Access. This article is licensed under a Creative Commons Attribution 4.0 International License, which permits use, sharing, adaptation, distribution and reproduction in any medium or format, as long as you give appropriate credit to the original author(s) and the source, provide a link to the Creative Commons licence, and indicate if changes were made. The images or other third party material in this article are included in the article's Creative Commons licence, unless indicated otherwise in a credit line to the material. If material is not included in the article's Creative Commons licence and your intended use is not permitted by statutory regulation or exceeds the permitted use, you will need to obtain permission directly from the copyright holder. To view a copy of this licence, visit http:// creativecommons.org/licenses/by/4.0/.

Publisher's Note Springer Nature remains neutral with regard to jurisdictional claims in published maps and institutional affiliations.

\section{References}

[1] Baylis, W.E. (ed.): Clifford (Geometric) Algebra with Applications to Physics, Mathematics, and Engineering. Birkhäuser, Boston (1996)

[2] Baylis, W.E.: Comment on 'Dirac theory in spacetime algebra'. J. Phys. A Math. Gen. 35, 4791-4796 (2002)

[3] de Beaucé, V., Sen, S., Sexton, J.C.: Chiral dirac fermions on the lattice using geometric discretisation. Nucl. Phys. B Proc. Suppl. 129-130, 468-470 (2004)

[4] Dezin, A.A.: Multidimensional Analysis and Discrete Models. CRC Press, Boca Raton (1995) 
[5] Faustino, N., Kähler, U., Sommen, F.: Discrete Dirac operators in Clifford analysis. Adv. Appl. Clifford Algebr. 17(3), 451-467 (2007)

[6] Faustino, N.: Solutions for the Klein-Gordon and Dirac equations on the lattice based on Chebyshev polynomials. Complex Anal. Oper. Theory 10(2), 379-399 (2016)

[7] Faustino, N.: A conformal group approach to the Dirac-Kahler system on the lattice. Math. Methods Appl. Sci. 40(11), 4118-4127 (2017)

[8] Hestenes, D.: Real spinor fields. J. Math. Phys. 8(4), 798-808 (1967)

[9] Hestenes, D.: Spacetime Algebra. Gordon and Breach, New York (1966)

[10] Joyce, W.P.: Dirac theory in spacetime algebra: I: the generalized bivector Dirac equation. J. Phys. A Math. Gen. 34, 1991-2005 (2001)

[11] Joyce, W.P., Martin, J.G.: Equivalence of Dirac formulations. J. Phys. A Math. Gen. 35, 4729-4736 (2002)

[12] Kähler, E.: Der innere differentialkül. Rendiconti di Matematica e delle sue Applicazioni 21(3-4), 425-523 (1962)

[13] Kanamori, I., Kawamoto, N.: Dirac-Kähler fermion from Clifford product with noncommutative differential form on a lattice. Int. J. Mod. Phys. A 19(5), 695-736 (2004)

[14] Rabin, J.M.: Homology theory of lattice fermion doubling. Nucl. Phys. B 201(2), 315-332 (1982)

[15] Sushch, V.: A discrete model of the Dirac-Kähler equation. Rep. Math. Phys. 73(1), 109-125 (2014)

[16] Sushch, V.: On the chirality of a discrete Dirac-Kähler equation. Rep. Math. Phys. 76(2), 179-196 (2015)

[17] Sushch, V.: A discrete Dirac-Kähler equation using a geometric discretisation scheme. Adv. Appl. Clifford Algebr. 28(72), 1-17 (2018)

[18] Sushch, V.: Discrete versions of some Dirac type equations and plane wave solutions. In: Pinelas, S., et al. (eds.) Differential and difference equations with applications, vol 230. Springer Proceedings in Mathematics and Statistics, pp 463-475. Springer, Cham (2018)

[19] Sushch, V.: Discrete Dirac-Kähler equation and its formulation in algebraic form. Pliska Stud. Math. 26, 225-238 (2016)

[20] Vaz, J.: Clifford-like calculus over lattice. Adv. Appl. Clifford Algebr. 7(1), 37-70 (1997)

Volodymyr Sushch

Koszalin University of Technology

Sniadeckich 2

75-453 Koszalin

Poland

e-mail: volodymyr.sushch@tu.koszalin.pl

Received: August 25, 2019.

Accepted: June 13, 2020. 